
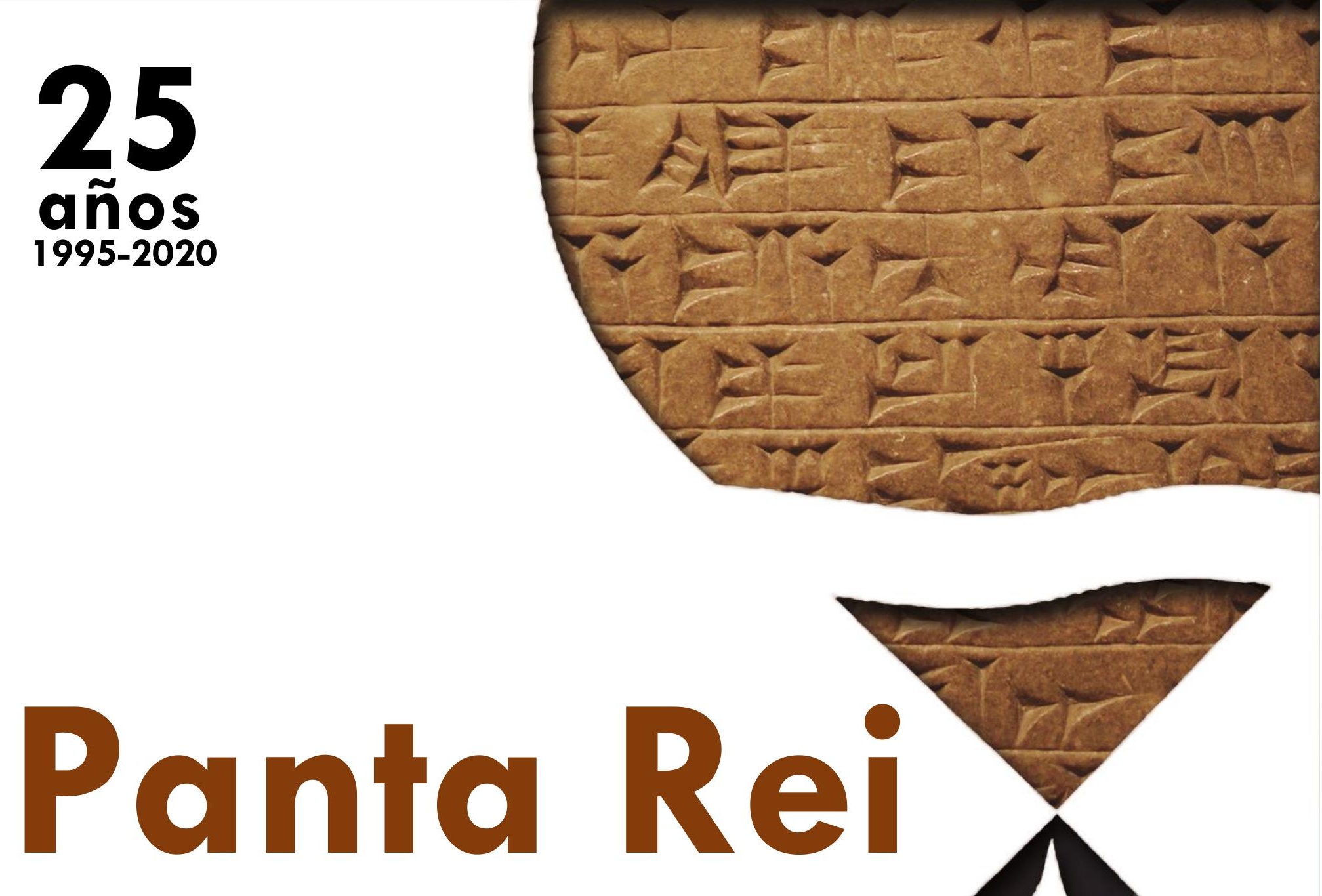

Revista digital de Historia y didáctica de la Historia 


\section{0 (2)}

Revista anual

Fecha de inicio: 1995

Revista Panta Rei. pantarei@um.es

\section{Edita:}

Centro de Estudios del Próximo Oriente y la Antigüedad Tardía - CEPOAT

Edificio Universitario Saavedra Fajardo.

Universidad de Murcia

C/ Actor Isidoro Máiquez, 9

30007 - MURCIA - ESPAÑA

Teléfono: $(+34) 868883890$

cepoat@um.es

Web: www.um.es/cepoat/pantarei

Ediciones de la Universidad de Murcia - EDITUM

Edificio Pleiades. Campus de Espinardo.

Universidad de Murcia

C/ Campus, s/n

30100 - MURCIA - ESPAÑA

Teléfono: $(+34) 868883013$

editum@um.es

Web: https://www.um.es/web/editum/

En portada: Montaje elaborado a partir de fotografía de una inscripción sumeria del British Museum (Wikimedia) (arriba) y fotografía de Miguel Martínez Sánchez (abajo)

Edición 2020 (2)

ISSNe: 2386-8864

ISSN: $1136-2464$
Responsables de los textos: sus autores.

Responsable de la presente edición: Consejo Editorial de Panta Rei.

Depósito legal: MU-966-1995 


\section{CONSEJO DE REDACCIÓN}

Coordinador editorial

Egea Vivancos, Alejandro [Didáctica de las Ciencias Sociales, Universidad de Murcia]

Secretaria

Arias Ferrer, Laura [Didáctica de las Ciencias

Sociales, Universidad de Murcia]

Editores

Jiménez Vialás, Helena [Historia Antigua, Universidad de Murcia]

Martínez Gil, Tània [Didáctica de las Ciencias

Sociales, Universidad de Barcelona]

Meseguer Gil, Antonio José [Historiador, Profesor de

Secundaria]

Ortiz García, Jónatan [Arqueología, Universidad de

Alcalá de Henares]

Romero Molero, Alberto [Arqueología, Universidad

Isabel I]

Sáez Giménez, David Omar [Historiador, Profesor

de Secundaria]

Sáez Rosenkranz, Isidora V. [Didáctica de las

Ciencias Sociales, Universidad de Barcelona]

Sánchez Mondejar, Celso Miguel [Arqueólogo,

Patrimonio Inteligente]

Responsable informático

Martínez García, José Javier [CEPOAT, Universidad de Murcia]

Responsables de traducción y corrección lingüística Martínez Martínez, Cristina [Profesora de Secundaria, Sociedad Española de Lenguas Modernas] Albaladejo Albaladejo, Sara [ISEN-Universidad de Murcia]

\section{CONSEJO ASESOR}

Adroher Auroux, Andrés María [Arqueología, Universidad de Granada]

Albero Muñoz, $M^{a}$ del Mar [H. ${ }^{a}$ del Arte, Universidad de Murcia]

Alia Miranda, Francisco [Historia Contemporánea, UCLM]

Arciniega García, Luis [Historia del Arte, Universidad de Valencia]

Barrio Barrio, Juan Antonio [Historia Medieval,

Universidad de Alicante]

Castellano i Solé, Núria [Egiptología, Schola

Didàctica Activa S.L.]

Chapman, Arthur [History Education, University

College of London, Reino Unido]

Cid López, Rosa María [Historia Antigua, Universidad de Oviedo]

Cobacho López, Ángel [Derecho, Universidad de Murcia]

Cuenca López, José María [Didáctica de las Ciencias Sociales, Universidad de Huelva]
Egea Bruno, Pedro M. ${ }^{a}$ [Historia Contemporánea, Universidad de Murcia]

Feijoo Martínez, Santiago [Arqueología, Consorcio

Ciudad Monumental de Mérida]

García Atienzar, Gabriel [Prehistoria, Universidad de

Alicante]

Ginestí Rosell, Anna [Filología Clásica, Katholische

Universität Eichstätt-Ingolstadt]

González Monfort, Neus [Didáctica de las

Ciencias Sociales, Universidad Autónoma de

Barcelona]

González Soutelo, Silvia [Arqueología, Universidad de Vigo]

Haber Uriarte, María [Prehistoria, Universidad de Murcia]

Hernández de la Fuente, David [Filología Clásica, Universidad Complutense]

Hutson, Scott R. [Anthropology, University of Kentucky, EEUU]

Igual Luis, David [Historia Medieval, UCLM]

Irigoyen López, Antonio [Historia Moderna,

Universidad de Murcia]

Jover Maestre, Francisco Javier [Prehistoria,

Universidad de Alicante]

Mahony, Simon [Digital Humanities, University College of London, Reino Unido]

Marsilla de Pascual, Francisco Reyes [Técnicas

historiográficas, Universidad de Murcia]

Martínez-Burgos García, Palma [H. ${ }^{a}$ del Arte, UCLM]

Mathis, Christian [Didaktik der Geschichte, PH Zürich]

Miralles Maldonado, José Carlos [Filología Clásica,

Universidad de Murcia]

Molina Gómez, José Antonio [Historia Antigua,

Universidad de Murcia]

Mónica Ghirardi [Historia Moderna, Universidad

Nacional de Córdoba, Argentina]

Navarro Espinach, Germán [Historia Medieval,

Universidad de Zaragoza]

Noguera Celdrán, José Miguel [Arqueología,

Universidad de Murcia]

Ortiz Heras, Manuel [Historia Contemporánea, UCLM]

Panzram, Sabine [Historia Antigua, Universität

Hamburg]

Pérez Molina, Miguel Emilio [Filología Clásica,

Universidad de Murcia]

Prados Martínez, Fernando [Arqueología,

Universidad de Alicante]

Sánchez lbáñez, Raquel [Didáctica de las Ciencias

Sociales, Universidad de Murcia]

Sancho Gómez, Miguel Pablo [Educación, UCAM]

Victoria Moreno, Diego [Historia Contemporánea,

UNED]

Vilar García, María José [Historia Contemporánea,

Universidad de Murcia]

Vivas Sainz, Inmaculada [H. ${ }^{a}$ del Arte, UNED]

Zamora López, José Ángel [Próximo Oriente Antiguo,

CCHS-CSIC] 



\section{Índice}

1995-2020. 25 años de Panta Rei

Equipo editorial

La Historia social aplicada a la antigua Mesopotamia: cambios historiográficos y nuevas vías de 9 investigación

Josué Javier Justel Vicente

A Look Back into Ancient Egyptian Linguistic Studies (c. 1995-2019)

Carlos Gracia Zamacona

La infancia en la antigua Mesopotamia: 25 años de investigación

Daniel Justel Vicente

Conflictos armados y su tratamiento en educación. Análisis de la producción científica de los últimos

25 años en la Web of Science

Naiara Vicent, Janire Castrillo, Alex Ibañez-Etxeberria y Leire Albas

La investigación en pensamiento histórico. Un estudio a través de las tesis doctorales de Ciencias

Sociales (1995-2020)

Álvaro Chaparro Sainz, M.a del Mar Felices de la Fuente y Laura Triviño Cabrera

Las narrativas nacionales en la enseñanza y el aprendizaje de la historia: Una revisión sobre los libros de texto y las narrativas de los estudiantes

César López Rodríguez

La historia de las personas: reflexiones desde la historiografía y de la didáctica de las ciencias sociales durante los últimos 25 años

Mariona Massip Sabater, Jordi Castellví Mata y Joan Pagès Blanch †

Panorámica de la trilogía cine, historia y educación en España (1995-2020) 



\title{
Las narrativas nacionales en la enseñanza y el aprendizaje de la historia: Una revisión sobre los libros de texto y las narrativas de los estudiantes
}

\author{
National Narratives in History Teaching and Learning: A Review on \\ History Textbooks and Students' Narratives
}

\author{
César López Rodríguez \\ Universidad Autónoma de Madrid \\ cesar.lopez@uam.es \\ 0000-0003-4616-8602
}

Recibido: 29/01/2020

Aceptado: 17/04/2020

\begin{abstract}
Resumen
Tradicionalmente, las narrativas nacionales han jugado un papel central en la enseñanza y el aprendizaje de la historia. Sin embargo, desde finales del siglo $X X$, existe un énfasis en señalar lo inadecuado de estas narrativas para el desarrollo del pensamiento histórico. Este artículo, mediante una revisión sistemática, analiza la evolución del papel de las narrativas nacionales en los últimos 25 años. Para ello, se analiza por un lado el papel de las narrativas nacionales en los manuales de historia. Por otro lado, se recogen los principales avances producidos en cómo los estudiantes narran la historia de su nación. Las investigaciones muestran una pervivencia de estas narrativas nacionales en la transmisión y comprensión del pasado, si bien continúan surgiendo proyectos relevantes para desnacionalizar la enseñanza del pasado. Finalmente, se discuten algunos de los retos futuros que se plantean para este tipo de investigaciones.
\end{abstract}

\section{Palabras clave}

Nacionalismo, Enseñanza de la Historia, Historia de Europa, Historiografía, Bibliografía.

\begin{abstract}
Traditionally, national narratives have played a central role in the teaching and learning of history. However, since the end of the 20th century, history educators have pointed out how these narratives can hinder the development of historical thinking. Through a systematic literature review, this article analyses the role of national narratives as it has evolved in the last 25 years. On the one hand, studies focused on the role of national narratives in history textbooks are analysed. On the other hand, the article reflects on the advances in research on how the students narrate the past of their nation. In general, research shows the relevance of these national narratives in the way the past is produced and consumed. Nevertheless, new projects continue to emerge which propose to denationalize the past. Finally, some of the new challenges for this line of research are discussed.
\end{abstract}

\section{Keywords}

Nationalism, History Instruction, European History, Historiography, Bibliographies.

Para citar este artículo: López Rodríguez, C. (2020). Las narrativas nacionales en la enseñanza y el aprendizaje de la historia: Una revisión sobre los libros de texto y las narrativas de los estudiantes. Panta Rei: revista digital de Historia y didáctica de la Historia, 149-166. doi: 10.6018/pantarei.445731 


\section{Introducción}

El surgimiento de la historia como disciplina moderna en los siglos XVIII y XIX estuvo marcado por un contexto fuertemente nacionalista. El principal objetivo de muchos historiadores y educadores, al servicio de los nuevos estados-nación que surgían en ese contexto, consistía en legitimar políticamente a la nación y fomentar una identidad nacional en sus ciudadanos (Berger, 2007; Berger y Lorenz, 2008; Lowenthal, 1998). Fundamentar esta legitimación política en el pasado, cuanto más remoto mejor, hizo de la disciplina de la historia una herramienta crucial para el desarrollo de la identidad nacional (Carretero, 2011; Smith, 1991). La propia historiografía romántica tradicional de cada nación produjo una narrativa nacional que recogía el largo recorrido de la nación desde su origen hasta la actualidad. Estas narrativas nacionales se constituyeron en narrativas oficiales y las escuelas en sus principales transmisores (Barton y Levstik, 2004; Hobsbawm, 1997; López, 2015; Wertsch, 2002).

El carácter legendario y mitológico de muchos de los contenidos de estas narrativas nacionales no suponía un obstáculo, ya que el principal objetivo no era realizar un análisis historiográfico crítico sobre el pasado, sino fomentar una identidad nacional y construir una memoria colectiva (López y Márquez, 2018; VanSledright, 2008; Wertsch, 2002). Como ya señalaba el famoso filósofo e historiador francés Ernest Renan (1882), el olvido, e incluso el error histórico, son un factor esencial en la creación de una nación. Una vez comenzado el siglo XX, y a lo largo del mismo, estas narrativas nacionales se constituyeron en verdades socialmente compartidas, raramente puestas a prueba 0 criticadas, que calcificaron en muchos países de Europa y sus colonias y en gran parte de Asia (Duara, 1995). La influencia de estas narrativas nacionales, tanto en el ámbito académico, como especialmente en el ámbito escolar y en los usos públicos del pasado, permanece hasta nuestros días.

En contraposición a este predominio de las narrativas nacionales y de los objetivos identitarios vinculados con la enseñanza de la historia, surge, a finales del siglo $X X$, un interés por entender la naturaleza misma del conocimiento histórico y los modos en el que este se produce y se consume (Lee, 2005; Seixas, 2004; Wineburg, 2001). Desde diferentes disciplinas como la propia historiografía, la sociología, las ciencias políticas o la psicología, se comienza a hacer hincapié en el carácter construido del conocimiento sobre el pasado. Las narrativas sobre el pasado dejan de ser consideradas como la historia en sí misma y pasan a verse más como herramientas a través de las que se reconstruye el pasado. Aparece un interés académico en conocer cómo se produce el conocimiento sobre el pasado y se presta un interés especial en conocer cómo los historiadores producen y estudian este pasado (Voss y Wiley, 2006). Términos como comprensión histórica o pensamiento histórico, que surgen en las últimas décadas, hacen referencia precisamente a aquello que hacen los expertos historiadores cuando tratan de entender el pasado. Esta manera de entender el conocimiento histórico surge en un momento de globalización y de supuesta crisis de los estados-nación, en el que se entiende que el fenómeno nacional ha quedado obsoleto. El papel del historiador se comienza a plantear como una tarea que dista mucho de reconocer nuestras raíces y nuestra identidad en nuestros antecesores, y se aproxima más a entender las enormes diferencias entre sociedades pasadas y presentes. El título de obras referentes en este sentido como Historical Thinking and Other Unnatural Acts (Wineburg, 2001) ० The Past is a Foreign Country (Lowenthal, 1985), enfatizan este distanciamiento entre el pasado y el presente. 
Como parece evidente, estos enfoques recientes sobre el pensamiento histórico suponen un fuerte desafío para las narrativas nacionales, que precisamente buscan establecer una línea de continuidad entre el pasado, el presente y el futuro del grupo. De hecho, uno de los objetivos que se plantean desde el desarrollo del pensamiento histórico es desmontar y desmitificar estas historias nacionales (Carretero, 2011).

El impacto de este cambio de paradigma en el estudio del pasado debería ser claro en el ámbito de la enseñanza y el aprendizaje de la historia. Esto parece plausible si tenemos en cuenta que dicho cambio se produce tanto en lo que se refiere a la propia naturaleza del conocimiento histórico, como a los objetivos que tiene el estudio del pasado. Por lo tanto, parece pertinente plantearse cómo este desarrollo producido en las últimas décadas ha impactado en el papel que juegan las narrativas nacionales en el aprendizaje y la enseñanza de la historia. A este respecto, consideramos muy pertinente utilizar la distinción planteada por Wertsch (1997) entre dos momentos clave de influencia de las narrativas sobre el conocimiento histórico: la producción y el consumo. La producción haría referencia a las narrativas y textos elaborados sobre el pasado y que, en el ámbito educativo, son transmitidos fundamentalmente a través de los libros de texto. El proceso de consumo hace referencia a cómo las personas finalmente entienden, usan o dan sentido a esas narrativas producidas (Wertsch, 1997). Ambos procesos son necesarios para entender cómo se transmite y cómo se comprende el pasado. Las personas no consumen pasivamente las narrativas que se producen sobre el pasado, pero tampoco generan sus propias narrativas de manera aislada sin recurrir a conocimientos socialmente compartidos. Por lo tanto, para entender el papel de las narrativas nacionales en la enseñanza y el aprendizaje de la historia en las últimas décadas, se hace necesario tener en cuenta los avances producidos en ambos procesos. En el presente artículo, a través de una revisión sistemática, se pretende dar respuesta a la siguiente pregunta: ¿̇cuál ha sido el papel de las narrativas nacionales en los procesos de producción y consumo del conocimiento histórico en los últimos 25 años? Para analizar los cambios en el proceso de producción de narrativas nacionales, nos centraremos en los estudios encaminados a examinar su papel en los libros de texto de historia. Esta elección está determinada por ser el libro de texto el mecanismo de producción que ha generado un mayor interés en el ámbito formal de la enseñanza de la historia, y cuya relevancia permanece hasta el presente. Por otro lado, analizaremos los principales estudios que se han llevado en los últimos 25 años para analizar cómo los estudiantes comprenden y generan sus propias narrativas sobre el pasado nacional.

\section{Méłodo}

La presente revisión pretende dar cuenta de los principales avances y cambios producidos en los últimos 25 años en torno a la presencia de las narrativas nacionales en los procesos de producción y consumo vinculados con la enseñanza y aprendizaje de la historia. En este sentido, en el ámbito de la producción, son múltiples los mecanismos que podrían analizarse -novelas históricas, lugares de memoria, museos o películas. Sin embargo, se ha decidido centrarse en la evolución de la presencia de las narrativas nacionales en los libros de texto, por ser estos un mecanismo de producción y transmisión central en la enseñanza formal de la historia y que más atención ha recibido en las últimas décadas. Para su análisis, hemos incluido en esta revisión aquellos trabajos más significativos producidos en las últimas décadas, sin cerrar el estudio a un ámbito nacional específico o a un nivel de educación concreto. Se intenta aquí dar una perspectiva amplia de cómo se ha desarrollado este ámbito en relación con los cambios ocurridos en la propia disciplina 
de la historia y la educación histórica hacia la construcción de un pensamiento histórico. Este mismo eje, la tensión entre el desarrollo del pensamiento histórico, por un lado, y la memoria colectiva por otro, es el que ha guiado el análisis de los estudios enfocados a entender cómo narran los estudiantes el pasado de su nación. La revisión pretende dar respuesta a qué mecanismos están involucrados en esta construcción, más que a indagar sobre qué saben o no los estudiantes sobre una determinada narrativa nacional.

Tanto para analizar los estudios centrados en los manuales de historia como en los estudiantes, se han priorizado los artículos científicos publicados en revistas con un alto índice de impacto, fundamentalmente aquellas recogidas en Web of Science y Scopus. Para la selección de trabajos se han utilizado ecuaciones de búsqueda con los descriptores en inglés, como national narratives o national history y textbooks o students y otros descriptores análogos. También se han incluido en esta revisión trabajos incluidos en libros y capítulos de libro de editoriales prestigiosas, así como trabajos derivados de proyectos de investigación centrados en la temática (e.g., Joint History Proyect, School Council Project (SHP) History 13-16 o Learning Each Other's Historical Narratives: Palestinian and Israeli Project). Finalmente, también se han tenido en cuenta los trabajos desarrollados en institutos o centros de investigación orientados específicamente al estudio de manuales y la enseñanza y aprendizaje de la historia, como EUROCLIO o el Georg Eckert Institute for International Textbook Research. A continuación, se muestran los principales resultados obtenidos relativos, por una parte, al papel de las narrativas nacionales en los manuales de historia y, por otra, a los estudios centrados en cómo los estudiantes construyen las narrativas sobre el pasado de su país.

\section{Resultados y discusión}

\subsection{Los libros de texto y las narrativas nacionales}

Desde las últimas décadas del siglo XX y hasta la actualidad, la enseñanza de la historia ha experimentado grandes cambios. Un catalizador fundamental de este cambio se produjo en Inglaterra a través del School Council Project (SHP) History 13-16, en la década de los 70 y 80. Este proyecto planteó la necesidad de abandonar la enseñanza de la historia basada en un enfoque nacionalista y centrada en transmitir eventos y fechas de un modo cronológico para promover un enfoque basado en el carácter construido de la historia y el papel activo del alumnado (Crawford, 2000; Dickinson, 2000; Foster, 1998; Hayden, 2004). Este cambio ponía un énfasis en los procesos de construcción de la historia y en cómo los historiadores construyen el conocimiento histórico. La oposición entre una "historia tradicional" nacionalista y una "nueva historia", más centrada en el cómo se produce el conocimiento histórico, constituye aún hoy una tensión no resuelta que ha tenido un gran impacto en las investigaciones sobre los libros de texto de historia y que guiará la presente sección (Carrier, 2018; Foster, 2011; Fuchs y Sammler, 2016).

En efecto, como comenta Foster (2012) para el caso inglés, la gran mayoría de libros de texto previos a 1970 presentaban una única narrativa nacionalista que reflejaba los logros de la nación, la época dorada del Imperio Británico y las acciones de los grandes monarcas. Este tipo de textos recogen las narrativas nacionales y las presentan como la Historia. Una única Historia con mayúscula, revestida de un carácter objetivo, ajena a la crítica y con un grado de autoridad irrefutable. El objetivo de este tipo de libros de texto es dar sentido al pasado nacionalizándolo 
y crear un vínculo entre el pasado y el presente centrado en la nación y la identidad nacional (Amin, 2014; Létourneau, 2017). Como señala Elie Podeh (2000) al hablar del caso israelí, los libros de texto constituyen el otro brazo del estado, que como una "corte suprema de la historia" deciden qué es apropiado incluir y qué no. De esta manera, los libros de texto han constituido una de las principales herramientas que influyen, e incluso controlan (Foster, 2012), lo que los jóvenes conocen del pasado de su nación. Alridge (2006), ponía de manifiesto cómo los libros de texto estadounidenses presentan una narrativa única, heroica y centrada en un ideal de progreso de la nación que niega a los estudiantes un conocimiento complejo y preciso de los acontecimientos históricos de su país. Jan Germen Janmaat (2005), indicaba cómo en los países de la antigua Unión Soviética los libros de texto proveen a los constructores del sentimiento nacional de una infinita fuente de materiales basados en mitos y leyendas que fundamentan el discurso nacionalista. En el caso de España, recientes análisis centrados en el papel de la narrativa nacional en los libros de texto (Gómez Carrasco y Molina Puche, 2017; Gómez Carrasco, Vivas Moreno, Miralles, 2019; Sáiz, 2017, 2018; Sánchez, Arias y Egea, 2016), muestran cómo existe una pervivencia de la narrativa nacional maestra centrada en los grandes hitos y personajes nacionales como la Reconquista, el Descubrimiento de América y el Imperio Español. No obstante, estos autores reconocen un giro hacia la integración de la narrativa nacional en el contexto europeo (Gómez Carrasco et al. 2019) y la inclusión de visiones más historiográficas sobre el pasado nacional. Pese a ello, los libros mantienen un contenido esencialista y nacional fundamentalmente a través de elementos formales, como el uso de etiquetas nacionales para referirse a períodos previos a la aparición de la nación misma o mediante imágenes icónicas de la pintura histórica romántica del siglo XIX (Sáiz, 2017). Además, estos estudios destacan el fuerte carácter memorístico de la enseñanza de la historia en España y el camino tradicionalista que ha seguido el currículo en los últimos 25 años.

Este enfoque nacionalista ha dado lugar a que los investigadores hayan encontrado visiones diferentes, cuando no contradictorias, sobre los mismos eventos históricos presentados en los libros de texto de diferentes naciones. Así, Crawford y Foster (2007), en su libro War, Nation, Memory, llevaron a cabo un minucioso análisis de cómo los libros de texto de China, Francia, Alemania, Japón, Estados Unidos y el Reino Unido, presentaban interpretaciones selectivas sobre la Segunda Guerra Mundial. Mientras que en China había un énfasis en la "masacre de Nanjing" cometida por el ejército japonés, los libros estadounidenses se centraban en las representaciones del ataque a Pearl Harbor o en la valentía y determinación mostradas por el pueblo estadounidense en el Día-D, la Victoria en Europa o la guerra en el Pacífico. Los textos británicos, por su parte, describían la victimización y el heroísmo del pueblo británico en la Batalla de Gran Bretaña, la evacuación de Dunkerque o el Blitz. De hecho, como comenta Foster (2012), cuando se trata de alguna guerra, los autores de los libros de texto en casi todas las naciones desarrollan narrativas que sirven a propósitos ideológicos y nacionales. Otro ejemplo en este sentido es el trabajo de Carretero, Jacott y López-Manjón (2002) sobre las representaciones del denominado "descubrimiento" de América en los libros de texto de México y España. Mientras que los libros de texto españoles enfatizaban los aspectos positivos del evento y el estatus superior de la cultura española, los libros de texto mexicanos resaltaban las consecuencias negativas de la llegada de los españoles y tomaban claramente partido por los nativos.

El papel de los libros de texto como transmisores de una narrativa nacional oficial marcadamente nacionalista, lejos de disminuir en las últimas décadas, parece acrecentarse (Carrier, 2018; Foster, 2012; Grever y Van der Vlies, 2017; Popp, 2009; Van der Leeuw-Roord, 2004). Como señalaba 
Stuart Foster (2012), en un análisis sobre el papel de los libros de texto en una época de globalización, la gran mayoría de libros de texto a lo largo del mundo comparten dos características principalmente: "a menudo adoptan una perspectiva nacionalista de los eventos históricos (...) y presentan a los estudiantes una única narrativa 'oficial"' (p. 51). Una variable fundamental a tener en cuenta al hablar de la pervivencia de las narrativas nacionales en los libros de texto es el control del estado sobre la educación. Como señala Susanne Popp (2009), "los puntos de referencia de la enseñanza de la historia han sido y siempre serán la identidad y la integración del sistema político que es responsable de esa educación" (p.112). Un famoso ejemplo del papel del estado fue la ley de 2005 en Francia que requería a los profesores de instituto a enseñar los 'valores positivos' del colonialismo francés a sus estudiantes. Grever y Van der Vlies (2017), en su reciente análisis de la pervivencia de las narrativas nacionales en los libros de texto, recogen cómo, en Rusia, el presidente Putin ha pedido a los historiadores desarrollar un currículo de historia que produzca una única historia libre de contradicciones y ambigüedades. En el mismo trabajo también recogen cómo en Estados Unidos muchos historiadores protestaron ante los cambios introducidos por el Panel de Educación de Tejas en los libros de texto en 2010, ya que muchos de esos cambios reflejaban básicamente objetivos políticos y eran historiográficamente incorrectos. Ejemplos similares han sido analizados en Japón (Bukh, 2007), Australia (Macintyre y Clark, 2003), Israel (Porat, 2001) o Grecia (Repoussi, 2006).

Frente a este predominio de las narrativas nacionales, en las últimas décadas han aparecido importantes ejemplos de libros de texto con un enfoque muy diferente. Una de las principales características de estos libros de texto es que no se presentan como los depositarios del pasado tal y como fue, sino que enfatizan el carácter construido e interpretativo del propio conocimiento histórico. Este cambio refleja también el giro en la enseñanza de la historia, que a finales de los 70 y principios de los 80 , enfatiza la distinción entre enseñar el pasado y pensar históricamente sobre el pasado (Dickinson, 2000; Hayden, 2004; Shemilt, 1980; Wineburg, 2001). Los libros de texto comenzaron a presentar las narrativas nacionales no como una verdad absoluta e indiscutible, sino como algo construido y abierto al debate y la crítica. Este enfoque disciplinar de la enseñanza de la historia se ve ejemplificado por el School Council Project (SHP) History 1316 desarrollado en Inglaterra y que, como comenta Foster (2012), tiene uno de sus más famosos ejemplos en el libro de Shepard, Reid y Shepherd (1993) Peace and War. Este libro de texto no está organizado en una narrativa lineal sobre la nación, sino que los capítulos hacen referencia a diferentes preguntas de investigación sobre las que reflexionar. El formato narrativo no es predominante, sino que diferentes fuentes como fotografías, periódicos o mapas, constituyen la base del libro. El lenguaje utilizado, lejos de ser autoritario e impersonal, muestra un carácter más condicional y tentativo, enfatizando frases como "algunos historiadores plantean...", que subrayan el carácter construido e interpretativo del conocimiento histórico. A menudo, en el libro, se utilizan diferentes fuentes que dan puntos de vista distintos sobre un mismo evento histórico, y las actividades no demandan un simple recuerdo de información, sino que buscan que el estudiante razone $\circ$ argumente sobre evidencias presentadas por las fuentes. Como puede observarse, la propia naturaleza del libro de texto se entiende de un modo distinto.

En esta línea, cabe destacar aquellos proyectos que han surgido en las últimas décadas y que presentan esfuerzos de autores de diferentes nacionalidades trabajando conjuntamente para desarrollar un libro de texto común (para una exhaustiva revisión de estos proyectos véase Maier, 2017). Ejemplos de estos proyectos comunes son el Manual de historia franco-alemán (Bernlochner y Geiss, 2006) o el proyecto ruso-alemán (Chubaryan y Maier, 2008), que aborda eventos como 
la Primera y la Segunda Guerra Mundial. Un conocido ejemplo que focaliza en las narrativas nacionales es el desarrollado bajo el Learning Each Other's Historical Narratives: Palestinian and Israeli Project (Adwan y Bar-On, 2007), que elaboró un libro de texto donde se recogían y confrontaban las narrativas nacionales israelí y palestina. El libro posee un formato a tres columnas, con cada narrativa nacional en un lateral y una columna central en blanco para las anotaciones, reflexiones e ideas de los estudiantes. Otro de los proyectos conjuntos más ambicioso es el Joint History Project (Koulouri, 2005), que integra 14 autores de 11 países del sureste europeo con la finalidad de plantear una alternativa inclusiva a las visiones etnocéntricas y las narrativas nacionales presentadas sobre la historia de los Balcanes en las diferentes naciones.

Como comenta Maier (2017), muchos de estos proyectos conjuntos tienen como finalidad promover la paz y la reconciliación de sociedades con un pasado reciente conflictivo. Asimismo, este autor enfatiza que la mayoría de estas empresas surgen de iniciativas vinculadas a ONGs y que dependen en gran medida de encontrar un contexto político adecuado. Efectivamente, aquellos países con un régimen democrático y un contexto político más inclusivo pueden favorecer la aparición de estos libros de texto que presentan una nueva manera de ver la historia y las narrativas nacionales (Popp, 2009). Por el contrario, como señalan Foster y Crawford (2006), en muchas naciones estado, bajo la idea del patriotismo, los jóvenes consumen la historia que sus líderes deciden y la enseñanza nacionalista y mono-cultural del pasado constituye el cemento para establecer la unidad nacional.

En todo caso, los estudios centrados en los libros de texto en las últimas décadas, ponen de manifiesto que no se trata de una cuestión de todo o nada, o de "buenos" libros de texto frente a "malos" (Grever y Van der Vlies, 2017). Tampoco parece que haya habido en las últimas tres décadas un giro claramente a favor de los nuevos enfoques disciplinares, ni mucho menos (Symcox y Wilschut, 2009). En parte, esto es debido a que optar por un enfoque centrado en la transmisión de narrativas nacionales $u$ otro centrado en el desarrollo del pensamiento histórico no depende únicamente de educadores o académicos, sino que cada vez más, estos debates incorporan agentes políticos y sociales. Fruto de estas tensiones y acalorados debates sobre cómo y qué se debe enseñar y recoger en los libros de texto, en las últimas décadas hemos asistido a las denominadas "guerras de la historia" (Fuchs, 2011 ; Macintyre y Clarck, 2003). Estos debates siguen girando en torno a aquellos que piensan que los jóvenes no tienen un conocimiento suficiente sobre la historia de su nación y aquellos que aspiran a desligar cada vez más el conocimiento histórico del conocimiento de las narrativas nacionales. Los estudios aquí recogidos muestran la aparición de iniciativas integradoras y enfocadas a hacer de los libros de texto una herramienta más para el desarrollo del pensamiento histórico. Sin embargo, lejos de constituir una mayoría, estas iniciativas continúan estando en desventaja con respecto a aquellas que promueven libros de texto al servicio de la construcción de la identidad nacional.

\subsection{Los estudiantes y las narrativas nacionales}

Como se ha señalado, desde posiciones conservadoras es frecuente encontrar encendidos debates sociales que reclaman que los jóvenes cada vez conocen menos la historia de su nación. Estas reclamaciones suelen lamentar la falta de conocimientos de los estudiantes sobre las grandes gestas nacionales o los grandes prohombres que han guiado a su nación a lo largo de la historia. En estas ocasiones, se identifica el conocimiento histórico con conocer una serie de eventos, fechas 
y personajes históricos. En definitiva, se demanda que los estudiantes aprendan una narrativa nacional.

Sin embargo, desde finales del siglo pasado, los investigadores del campo de la enseñanza de la historia han puesto el énfasis en enseñar a los estudiantes a pensar históricamente (Lee, 2005; Seixas, 2004). Para ello, se han postulado una serie de habilidades cognitivas que tienen que ver no tanto con el qué de la historia, sino con el cómo se construye el conocimiento histórico. Habilidades como el desarrollo de la empatía histórica, la multiperspectividad, entender el cambio y la continuidad o saber evaluar fuentes históricas, constituyen alguno de los objetivos establecidos para los estudiantes. Asimismo, no se abandona el conocimiento de conceptos de carácter declarativo centrales para el conocimiento histórico, por lo que también se postula que los estudiantes deben comprender conceptos como "democracia", "revolución", "absolutismo" o "socialismo" (Rodríguez Moneo y López, 2017; Voss y Wiley, 2006).

Si nos centramos en el aprendizaje de las narrativas nacionales, desde el enfoque tradicional, los estudiantes deberían memorizar las narrativas nacionales, conocer los grandes eventos y personajes de su país, desarrollar una identificación con ellos y adquirir una serie de valores morales propios de su identidad nacional. En contraposición, desde el enfoque disciplinar, los estudiantes deberían entender las narrativas nacionales como una construcción histórica y no como la historia en sí misma. También se fomentaría tener en cuenta diferentes narrativas que reflejen una multiperspectividad sobre el pasado y la evaluación de esas narrativas como fuentes históricas. En cuanto a los conceptos centrales presentes en las narrativas nacionales, no cabe duda que el propio concepto de nación o de identidad nacional constituyen conceptos vertebradores de estas narrativas (Rodríguez Moneo y López, 2017). Por tanto, adquirir un conocimiento historiográfico sobre estos conceptos ayudaría a comprender históricamente las narrativas nacionales.

En los últimos años han crecido las investigaciones sobre cómo los estudiantes comprenden estos conceptos y elaboran sus propias narrativas (López, Carretero y Rodríguez-Moneo, 2014a, 2014b). Diversos estudios subrayan que, en su mayoría, los estudiantes entienden dichos conceptos de un modo esencialista y nacionalista. En varios trabajos realizados con estudiantes españoles, se ha puesto de manifiesto cómo la nación y la identidad nacional se constituyen en protagonistas atemporales de la historia (Egea Vivancos y Arias Ferrer, 2015; López et al. 2015; López, 2019; Sáiz, 2017; Sáiz y Barca, 2019). Los estudiantes suelen erigir a la nación como el sujeto en torno al cual se desarrollan sus narrativas, protagonizando incluso eventos previos al surgimiento de la nación misma. Así, pese a que la gran mayoría de los historiadores entienden las naciones como una construcción social de carácter moderno, los estudiantes tienden a pensar que han existido desde siempre. De este modo, la nación protagoniza eventos ocurridos en períodos como la Edad Media o incluso anteriores. A modo de ejemplo, varios estudios muestran cómo estudiantes españoles entienden que, durante la denominada Reconquista, España fue conquistada por los musulmanes y posteriormente fueron los propios españoles los que terminan reconquistando España (López et al. 2015; Sáiz, 2017). Este modo de entender el concepto de nación no parece ni mucho menos algo exclusivo de los españoles. También se ha encontrado un predominio de estas concepciones esencialistas en estudios realizados en América Latina (Carretero, López, González y Rodríguez-Moneo, 2012). Además, no parece que sea algo restringido al modo de entender la propia nación. En un estudio realizado con estudiantes 
españoles sobre la narrativa nacional griega, los participantes mayoritariamente reflejaban una comprensión atemporal y estática de dicha nación (López et al., 2014a).

Otra de las principales conclusiones a las que llegan los estudios realizados con estudiantes es que suelen establecer una identificación con el pasado en términos nacionales. Es decir, es frecuente que utilicen términos como "nosotros" o "nuestro" para referirse a acontecimientos ocurridos mucho antes de su nacimiento e incluso antes de la aparición de su nación (Barton y Levstik, 2004; López y Márquez, 2018). Por ejemplo, en los estudios llevados a cabo sobre la denominada Reconquista es fácil encontrar identificaciones como la siguiente: "La Batalla de Las Navas de Tolosa fue una batalla muy importante que les ganamos. Al final, les echamos de Granada en 1492." (López, et. al. 2015, p.13).

La identificación nacional con los personajes del pasado contribuye de manera determinante a que los estudiantes narren el pasado en términos de nosotros y ellos. Esta simplificación de la narrativa, que homogeniza y olvida a infinidad de grupos bajo la etiqueta nacional, tiene un fuerte impacto no sólo en el modo en el que los individuos piensan sobre el pasado, sino también en cómo lo evalúan y cómo lo sienten. Varios trabajos han encontrado que los estudiantes suelen evaluar positivamente las acciones de su nación frente a las acciones de otros grupos (Bilali, 2013; Doosje y Branscombe, 2003; Lévesque, 2017; López et al. 2015). Doosje y Branscombe (2003), en un estudio realizado con estudiantes holandeses, mostraron cómo estos tendían a dar explicaciones basadas en factores externos para justificar las acciones negativas cometidas por su nación en Indonesia. Sin embargo, al evaluar las acciones cometidas por los alemanes durante la ocupación alemana de los Países Bajos, los estudiantes holandeses atribuyeron esas acciones a factores internos, personales, de los alemanes. Por su parte, Bilali (2013) en un trabajo que analizaba el papel de los turcos en el genocidio armenio ocurrido después de la Primera Guerra Mundial, encontró cómo los estudiantes turcos minimizaron la responsabilidad de su propio grupo nacional en el proceso. Los estudiantes turcos elaboraban estrategias de exoneración argumentando que ambos grupos fueron agresores o incluso que los turcos fueron las víctimas del conflicto. Por su parte, Lévesque (2017), muestra cómo los estudiantes canadienses más identificados con su comunidad histórica fueron los que produjeron las más fervientes y militantes narrativas sobre el pasado de su nación, utilizando frecuentemente la primera persona del plural en sus narrativas.

Estos estudios muestran en su conjunto cómo los estudiantes suelen construir narrativas simplificadas de conflictos entre el nosotros nacional y el ellos extranjero. Ya sean españoles contra árabes en la Reconquista (López et. al 2015), turcos contra armenios en el siglo XX (Bilali, 2013) o franceses contra ingleses (Lévesque, 2017), las narrativas de los estudiantes suelen presentar esos conflictos como luchas entre grupos monolíticos, que permite una identificación nacional con el pasado. Los estudiantes establecen estas vinculaciones entre su propia identidad y aquellos protagonistas del pasado remoto porque entienden que existe una continuidad fundamentada en la común identidad nacional. No toman conciencia de la distancia que separa esas dos sociedades - la del pasado y la del presente - y no parecen usar esa perspectiva histórica que caracteriza el modo de pensar de los historiadores.

Los procesos de identificación nacional con los actores del pasado tienen también una gran influencia en las emociones que experimentan los estudiantes (Doosje et al., 1998; László, 2014; Rüsen, 2004). La vinculación nacional permite experimentar lo que se ha denominado como 
emociones basadas en el grupo. Es decir, los estudiantes experimentan emociones en primera persona como el orgullo, la culpa o la vergüenza, con respecto a acciones llevadas a cabo por su grupo nacional, aunque estas acciones tuvieran lugar siglos antes de su nacimiento. Por ejemplo, en un estudio llevado a cabo con estudiantes holandeses sobre el pasado colonial de su nación (Doosje et al., 1998), aparecieron frecuentemente emociones de culpa colectiva por las acciones cometidas durante la colonización de Indonesia. De manera interesante, esta experiencia de culpa estaba mediada por el nivel de identificación con la nación. Así, aquellos participantes con una alta identificación con la nación focalizaban más en argumentos positivos respecto a su nación y no experimentaban esos sentimientos de culpa colectiva. Estos estudios centrados en las emociones que experimentan los estudiantes sobre el pasado nacional, si bien son hasta la fecha poco frecuentes, parecen ser muy relevantes si queremos entender mejor los factores que influyen en cómo nuestros estudiantes narran el pasado. Por ejemplo, existen numerosos países con un pasado problemático, como puede ser el caso de aquellos con un pasado colonial, en los que la historia nacional está fuertemente influida por sentimientos como la culpa o la vergüenza (Doosje et al. 1998; López y Márquez, 2018, 2020; Rüsen, 2004).

Si tenemos en cuenta los resultados de los estudios realizados en las últimas décadas centrados en comprender cómo construyen los estudiantes el pasado de su nación, parece claro que existe un predominio de las narrativas nacionales maestras (Barton y Levstik, 2004; Bilali, 2013; Carretero y Van Alphen, 2014; López, 2019; Olofsson, Samuelsson, Stolare y Wendell, 2017; Sáiz y López Facal, 2016). Hemos señalado cómo, por ejemplo, en el caso de España, la narrativa nacional de la Reconquista sigue fuertemente presente en la mente de los estudiantes. En países como estados Unidos, las narrativas sobre el pasado de la nación reflejan una perenne lucha por la libertad y el progreso (Barton y Levstik, 2004). En Suecia, los estudiantes narran la historia de su país como un país excepcional, ejemplo de paz, progreso, democracia y prosperidad (Olofsson et al., 2017). Además, si tenemos en cuenta los resultados de las diferentes investigaciones, este modo de narrar el pasado no parece estar muy influenciado por la edad de los participantes (Carretero y Van Alphen, 2014). Incluso existe una fuerte presencia de estas narrativas nacionales en futuros profesores de ciencias sociales (Sáiz y López Facal, 2016).

Por lo tanto, pese al énfasis puesto en la enseñanza de la historia en las últimas décadas en el desarrollo de un pensamiento histórico, las narrativas nacionales siguen teniendo un gran peso en el modo en el que las personas entienden y construyen el pasado. El conocimiento de los estudiantes parece tener más que ver con la construcción de una memoria colectiva, con unos objetivos de tipo identitarios, que con una visión disciplinar del pasado. En el mejor caso, como al hablar de los libros de texto, parece que nuevamente nos encontramos con una tensión entre ambos objetivos en lo que se refiere a la enseñanza que reciben los estudiantes. Como señala James Werstch (2017):

La enseñanza de la historia bien podría denominarse enseñanza de la memoria y la historia, porque está generalmente enfocada tanto a inculcar un relato del pasado que permite a las naciones reproducirse a sí mismas como "comunidades mnemónicas" y también a alentar a los estudiantes a adquirir una perspectiva crítica en los materiales y textos que usan para acceder al pasado (p.153).

Para entender mejor por qué se produce esta pervivencia de las narrativas nacionales en la mente de los individuos, parece necesario que se continúe realizando una investigación que se centre en cómo y por qué los estudiantes narran de este modo el pasado y no tanto en el contenido 
de sus narrativas. Una aportación que ha sido clave en este sentido ha sido la noción de esquema narrativo propuesta por Werstsch (1997). Esta noción hace referencia a cómo las narrativas que generan los individuos están influenciadas por patrones o esquemas narrativos más generales desarrollados y aprendidos a un nivel social o grupal. Estos esquemas narrativos constituyen un saber compartido por los miembros de un determinado grupo y son transmitidos tanto en ámbitos formales -fundamentalmente a través de la enseñanza de la historia - como informales - en películas, museos, novelas históricas o medios de comunicación. Estos esquemas tienen un fuerte impacto en la construcción de la identidad colectiva de los grupos y sirven de guía a las narrativas que emplean los individuos. Un ejemplo de este tipo de esquemas es el denominado triunfo contra fuerzas extranjeras (Wertsch, 2002). Wertsch encontró cómo diferentes narrativas del pasado de Rusia, como las invasiones mongolas del siglo XIII, la invasión napoleónica o la invasión alemana durante la Segunda Guerra Mundial, seguían siempre un mismo patrón o esquema narrativo. Este esquema narrativo consiste en el inicio de la agresión por parte de una fuerza extranjera, seguido de un período de sufrimiento por parte del pueblo ruso, que termina finalmente expulsando a las fuerzas extranjeras de su territorio. Este es un patrón que refleja el carácter irreductible y heroico del pueblo ruso a lo largo de la historia y que conforma su identidad nacional. Este mismo esquema es el que encontramos, por ejemplo, en el modo en que se narra la denominada Reconquista en España o la denominada Ocupación otomana de Grecia entre otros (López y Márquez, 2018). Recientemente, algunas investigaciones están poniendo de manifiesto el papel que juegan estos esquemas narrativos en el modo en que los estudiantes recuerdan el pasado (López, 2019; Wills, 2011). El uso de estos esquemas, los procesos de identificación grupal y emocional en términos nacionales y la comprensión esencialista de conceptos claves como los de nación o identidad nacional, parecen constituir las bases de la pervivencia de las narrativas nacionales en la mente de los estudiantes.

\section{Conclusiones y retos futuros}

Desde finales del siglo pasado, el campo de la enseñanza de la historia ha experimentado un innegable cambio hacia el desarrollo del pensamiento histórico (Lee, 2005; Seixas, 2004; Wineburg, 2001). La globalización, el auge de las migraciones y la aparente crisis del fenómeno nacional contribuyeron a que la propia disciplina histórica comenzara a plantearse la viabilidad y la validez de las tradicionales narrativas nacionales (Schissler y Soysal, 2005). Sin embargo, parece que la tensión entre los enfoques más centrados construir una memoria colectiva y aquellos que apuestan por fomentar un pensamiento histórico está lejos de resolverse. A través de los estudios recogidos en este artículo, queda de manifiesto la pervivencia de las narrativas nacionales tanto en los manuales de historia como, sobre todo, en la mente del alumnado.

Las investigaciones en torno a los libros de texto realizadas en las últimas décadas muestran la pervivencia de las narrativas nacionales en la enseñanza de la historia (Foster, 2012; Grever y Van der Vlies, 2017). El carácter narrativo, junto con un claro predominio de los contenidos nacionales, parecen ser una constante en muchos países a lo largo del mundo. El libro de texto continúa siendo uno de los principales transmisores de las narrativas nacionales. Sin embargo, en los últimos 25 años hemos asistido a un creciente esfuerzo por parte de proyectos que tratan de plantear de un modo distinto los manuales de historia. Un modo que presenta al manual no como el depositario de la verdadera historia, sino como una herramienta más para interpretar el pasado. Una herramienta abierta a la crítica y que incluye diferentes narrativas sobre el pasado. A lo largo del presente artículo se han mostrado ejemplos de manuales que tratan de romper con 
el formato narrativo nacional para centrarse en desarrollar habilidades del pensamiento histórico. Sin embargo, estos manuales no están exentos de dificultades. Por ejemplo, al plantear un enfoque más centrado en entender el proceso de construcción del pasado, se corre el riesgo de trabajar contenidos aislados y fragmentados, que dificulten elaborar un contexto temporal coherente. Asimismo, en ocasiones, aunque no se presente una narrativa lineal de la evolución del país, muchos de los contenidos seleccionados para trabajar habilidades históricas, como la empatía, la evaluación de fuentes o los juicios morales, continúan centrados en el ámbito nacional.

En las últimas décadas han surgido también con fuerza manuales fruto del trabajo conjunto de autores de diferentes nacionalidades (Adwan y Bar-Om, 2007; Maier, 2017). Estos manuales constituyen un esfuerzo para fomentar los procesos de paz y reconciliación entre naciones con un pasado conflictivo. Sin embargo, como se ha señalado, estos manuales necesitan del contexto político adecuado para su desarrollo. Un contexto político que, salvo algunas excepciones, parece estar más en la línea de fomentar visiones nacionalistas sobre el pasado.

Uno de los retos fundamentales con los que se enfrentan las nuevas investigaciones sobre los manuales escolares consiste en el cambio de la propia naturaleza del libro de texto. Este está pasando de ser meramente un libro de texto a incluir, cada vez más, enlaces a materiales multimedia. Como han señalado varios de los trabajos analizados, los estudios recientes cada vez se centran más en estudiar el libro de texto como un material complejo, en el que se deben analizar, no solo los textos, sino también las actividades que se proponen, el uso de las imágenes que se incluyen en él o el papel que juegan los mapas históricos (Grever y Van der Vlies, 2017; Sáiz, 2017). Estos contenidos, pese a que no tengan un formato narrativo, en numerosas ocasiones refuerzan de una manera implícita las grandes narrativas nacionales. La inclusión en las aulas de materiales diferentes al libro de texto, como pueden ser vídeos, páginas web o fuentes de información sobre el pasado accesibles vía internet, constituyen una buena oportunidad para poner a prueba el carácter autoritario e incuestionable del libro de texto.

Pese a algunos de los avances señalados en relación a los libros de texto, las investigaciones centradas en cómo los estudiantes comprenden el pasado de su nación, muestran un predominio claro de las narrativas nacionales (López et al. 2015; Olofsson et al. 2017; Sáiz, 2018; Sáiz y Barca, 2019; Sánchez et al., 2016). Más que haber adquirido unas habilidades que les permitan entender el carácter construido de la historia, tomar una perspectiva histórica para diferenciar el pasado del presente o elaborar juicios morales poniéndose en un contexto diferente al suyo, las investigaciones parecen apuntar claramente a una visión nacionalista del pasado que reconstruye la narrativa nacional. Los estudiantes suelen alcanzar objetivos de tipo más bien identitario que de carácter historiográfico.

Los esfuerzos de las investigaciones durante las últimas décadas se han centrado en entender por qué se produce esta comprensión nacionalizada del pasado. Es decir, no tratan tanto de evaluar qué elementos de la narrativa nacional conocen o no, sino qué aspectos son fundamentales para entender cómo narran el pasado. Los avances producidos en el propio campo de la historiografía, la enseñanza de la historia y otras disciplinas como la psicología o la sociología, han sido fundamentales a la hora de entender esta construcción. Así, el importante papel que juegan conceptos históricos como el de nación o identidad nacional en la construcción de las narrativas ha sido puesto de manifiesto (Rodríguez Moneo y López, 2017). Parece claro que una comprensión esencialista y romántica de estos conceptos vertebra las narrativas de los 
estudiantes. Del mismo modo, los procesos de identificación grupal están muy presentes en el modo en el que los estudiantes ven el pasado (Bilali, 2013; López y Márquez, 2018). Además, este proceso de identificación impacta en el tipo de juicios morales y emocionales que llevan a cabo (Doosje et al. 1998; López y Márquez, 2020).

Independientemente del uso que se haga del formato narrativo en la enseñanza, lo cierto es que este formato parece predominante en el modo en el que las personas damos sentido al pasado. Las aportaciones centradas en entender las herramientas culturales mediadoras para la construcción de las narrativas han supuesto un gran avance para entender cómo los individuos narran el pasado. La noción de esquema narrativo es un elemento clave para entender mejor la vinculación entre la memoria del individuo y la memoria colectiva (Wertsch, 1997, 2002). Las narrativas nacionales se perpetúan a través de estos esquemas narrativos que son compartidos socialmente dentro del grupo nacional. Estos esquemas se transmiten tanto dentro del ámbito formal como del informal. Este hecho podría ayudar a explicar la aparente contradicción entre los avances producidos en la elaboración de los manuales de historia y las narrativas de los estudiantes. En ocasiones, los estudiantes producen una narrativa nacional mucho más romántica que la que encontramos en los libros de texto en la actualidad (López, 2019). La influencia de los esquemas narrativos presentes en el ámbito informal puede estar en la base de este fenómeno. Incorporar investigaciones centradas en el ámbito informal aparece como uno de los grandes retos para comprender mejor las narrativas de nuestros estudiantes.

Cada vez más, los debates sobre el pasado están rebasando los límites de las aulas y los libros de texto. El papel de estos como principales transmisores del conocimiento sobre el pasado nacional disminuye a medida que aparecen nuevos entornos de transmisión del conocimiento histórico. Las redes sociales, como Facebook o Twitter, se están constituyendo en medios de masas en los que se discute el pasado. Los periódicos digitales, las plataformas de contenidos online o el aumento del fenómeno de las recreaciones históricas, son algunos de los nuevos mecanismos a través de los cuales los individuos reciben información sobre el pasado. Incorporar estos nuevos mecanismos de producción y consumo constituye, sin duda, uno de los retos actuales para entender cómo las personas construyen las narrativas sobre el pasado de su nación.

\section{Agradecimientos}

La elaboración de este trabajo se ha realizado bajo la ayuda del Ministerio de Ciencia, Innovación y Universidades con código RTI2018-096495-B-I00.

\section{Bibliografía}

Adwan, S. y Bar-On, D. (2007). Leading forward: The experiences of Palestinians and Israelis in the Learning Each Other's Historical Narratives project. En L. Cajani (ed.), History teaching, identities and citizenship (pp. 143-166). Trent, Reino Unido: Trentham Books.

Alridge, D. P. (2006). The limits of master narratives in history textbooks: An analysis of representations of Martin Luther King. Jr. Teachers College Record, 108, 662-686. doi: 10.1111/i.1467-9620.2006.00664.x

Amin, S.N. (2014). The impact of identity politics in challenging national narratives: A case study among Canadian Muslims. Studies in Ethnicity and Nationalism, 14(3), 418-35. doi: $10.1111 /$ sena.12105 
Barton, K. C. y Levstik, L. (eds.) (2004). Teaching History for the Common Good. Mahwah, NJ: Lawrence Erlbaum Associates, Inc.

Berger, S. (2007). Writing national histories in Europe: Reflections on the pasts, presents, and futures of a tradition. En K. H. Jarausch y T. Lindenberger (eds.), Conflicted Memories: Europeanizing contemporary histories (pp.22-68). Nueva York: Berghahn Books.

Berger, S. y Lorenz, C. (eds.) (2008). The Contested Nation: Ethnicity, Class, Religion and Gender in National Histories. Basingstoke: Palgrave Macmillan.

Bilali, R. (2013). National narrative and social psychological influences in Turks' denial of the mass killings of Armenians as genocide. Journal of Social Issues, 69(1), 16-33. doi: $10.1111 /$ iosi.12001

Bukh, A. (2007). Japan's history textbooks debate: National identity in narratives of victimhood and victimization. Asian Survey, 47(5), 683-704. doi: 10.1525/as.2007.47.5.683

Carretero, M. (2011). Constructing patriotism. Teaching of history and memories in global worlds. Charlotte, CT: Information Age Publishing.

Carretero, M. y Van Alphen, F. (2014). Do master narratives change among high school students? A characterization of how national history is represented. Cognition and Instruction, 32(3), 290-31 2. doi: $10.1080 / 07370008.2014 .919298$

Carretero, M., Jacott, L. y López-Manión, A. (2002). Learning history through textbooks: are Mexican and Spanish students taught the same story? Learning and Instruction, 12(6), 651 665. doi: $10.1016 / 50959-4752(01) 00036-6$

Carretero, M., López, C., Fernanda, M. F. y Rodríguez-Moneo, M. (2012). Students historical narratives and concepts about the nation. En M. Carretero, M. Asensio y M. RodríguezMoneo (eds.), History Education and the Construction of National Identities (pp. 153-170). Charlotte CT: Information Age Publishing.

Carrier, P. (2018). The nation, nationhood, and nationalism in textbook research from 1951 to 2017. En E. Fuchs y A. Bock (eds.), The Palgrave Handbook of Textbook Studies (pp. 181 198). Londres: Palgrave Macmillan.

Chubaryan, A. y Maier, R. (2008). Twentieth Century History of Germany in a New Setting: Sources, Statistics, Art Documents. Moskva: Olma.

Crawford, K. (2000). Researching the ideological and political role of the history textbook issues and methods. History Education Research Journal, 1 (1), 55-62. doi: 10.18546/HERJ.01.1.07

Crawford, K. A. y Foster, S. J. (2007). War Nation Memory: International Perspectives on World War II in School History Textbooks. Greenwich, CT: Information Age Publishing.

Dickinson, A. (2000). What should history be? En A. Kent (ed.), School subject teaching: The history and future of the curriculum. Londres: Kogan Page.

Doosje, B. y Branscombe, N. R. (2003). Attributions for the negative historical actions of a group. European Journal of Social Psychology, 33(2), 235-248. doi: 10.1002/ejsp. 142

Doosje, B., Branscombe, N. R., Spears, R. y Manstead, A. S. R. (1998). Guilty by association: when one's group has a negative history. Journal of Personality and Social Psychology, 75, 872886. doi: $10.1037 / 0022-3514.75 .4 .872$ 
Duara, P. (1995). Rescuing History from the Nation: Questioning Narratives of Modern China. Chicago: University of Chicago Press.

Egea Vivancos, A. y Arias Ferrer, L. (2015). Russell Crowe el gladiador, Colón el descubridor y otros protagonistas de la Historia. Buceando en las narrativas históricas de jóvenes universitarios españoles. CLIO. History and History teaching, 41. https://dialnet.unirioja.es/servlet/articulo? codigo $=5303982$

Foster, S. J. (1998). Politics, parallels and perennial curriculum questions: the battle over school history in England and the United States. Curriculum journal, 9(2), 153-164. doi: $10.1080 / 0958517970090203$

Foster, S. J. (201 1). Dominant traditions in international textbook research and revision. Education Inquiry, 2(1), 5-20. doi: 10.3402/edui.v2i1.21959

Foster, S. J. (2012). Re-thinking historical textbooks in a globalised world. En M. Carretero, M. Asensio y M. Rodríguez-Moneo (eds.), History Education and the Construction of National Identities (pp. 49-62). Charlotte CT: Information Age Publishing.

Foster, S. J. y Crawford, K. A. (eds.). (2006). What Shall We Tell the Children? International Perspectives on School History Textbooks. Greenwich, CT: Information Age Publishing.

Fuchs, E. (2011). Current trends in history and social studies textbook research. Journal of International Cooperation in Education, 14(2), 17-34. doi: 10.15027/34395

Fuchs, E. y Sammler, S. (2016). Textbooks between Tradition and Innovation: A journey through the history of the Georg Eckert Institute. Braunschweig: Georg Eckert Institute.

Geiss, P., Le Quintrec, G., y Bernlochner, L. (2006). Histoire/Geschichte: Europa und die Welt seit 1945. Stuttgart: Klett.

Gómez, C. J. y Puche, S. M. (2017). Narrativas nacionales y pensamiento histórico en los libros de texto de Educación Secundaria de España y Francia. Análisis a partir del tratamiento de los contenidos de la Edad Moderna. Vínculos de Historia, 6, 206-229. doi: $10.18239 / \mathrm{vdh} . \mathrm{V} 0 \mathrm{ib} .011$

Gómez, C. J., Moreno, V. V. y Martínez, P. M. (2019). Competencias históricas y narrativas europeas/nacionales en los libros de texto. Cadernos de Pesquisa, 49(172), 210-234. doi: $10.1590 / 198053145406$

Grever, M. y Van der Vlies, T. (2017). Why national narratives are perpetuated: A literature review on new insights from history textbook research. London Review of Education, 15(2), 286-301. doi: 10.18546/LRE.15.2.11

Haydn, T. (2004). History. En J. White (ed.), Rethinking the school curriculum (pp.87-103). Londres: Routledge.

Hobsbawm, E. (1997). Nations and Nationalism since 1780: programme, myth, reality. Cambridge: Cambridge University Press.

Janmaat, J. G. (2005). Ethnic and Civic Conceptions of the Nation in Ukraine's History Textbooks. European Education, 37(3), 20-37. doi: 10.1080/10564934.2005.11042394

Koulouri, C. (2005). Teaching Modern South East European History. Alternative Educational Materials, vols. 1-4. Thessaloniki: Centre for Democracy and Reconciliation in South East Europe (CDRSEE). 
László, J. (2014) Historical Tales and National Identity: An introduction to narrative social psychology. Londres: Routledge.

Lee, P. (2005). Putting principles into practice: understanding history. En M. S. Donovan y J. D. Bransford (eds.), How students learn: history, mathematics and sciences in the classroom? (pp.31-78). Washington: National Academies Press.

Létourneau, J. (2017). Teaching national history to young people today. En M. Carretero, S. Berger y M. Grever (eds.), Palgrave Handbook of Research in Historical Culture and Education (pp.227-42). Basingstoke: Palgrave Macmillan.

Lévesque, S. (2017). History as a "GPS": On the uses of historical narrative for French Canadian students' life orientation and identity. London Review of Education, 15(2), 227-242. doi: 10.18546/LRE.15.2.07

López, C. (2015). Repensando las narrativas nacionales: Un análisis del origen, transmisión e influencia en el aprendizaje histórico. Panta Rei. Revista Digital de Ciencia y Didáctica de la Historia, 77-92. doi: 10.6018/pantarei/2015/6

López, C. (2019). When 'we'are the alien forces: Misremembering the Conquest of America through the 'Triumph over alien forces' schematic narrative template. Culture \& Psychology. doi: $10.1177 / 1354067 \times 19894937$

López, C., Carretero, M. y Rodríguez-Moneo, M. (2014a). Telling a national narrative that is not your own. Does it enable critical historical consumption? Culture \& Psychology, 20(4), 547571. doi: $10.1177 / 1354067 \times 14554156$

López, C., Carretero, M. y Rodríguez-Moneo, M. (2014b). Fostering National Identity, Hindering Historical Understanding. En The Catalyzing Mind (pp. 21 1-221). Nueva York, NY.: Springer.

López, C., Carretero, M. y Rodríguez-Moneo, M. (2015). Conquest or Reconquest? Students' Conceptions of Nation Embedded in a Historical Narrative. Journal of the Learning Sciences, 24(2), 252-285. doi: 10.1080/10508406.2014.919863

López, C. y Márquez, M. G. (2018). Aportaciones de la psicología a la comprensión de las narrativas nacionales y la identidad nacional: pensamiento narrativo, identidades sociales y emociones colectivas. En A. Delgado, y A. Rivera (eds.), ¿̇Qué saben de su historia nuestros jóvenes? Enseñanza de la historia e Identidad nacional (pp. 99-1 18). Granada: Comares.

López, C. y Márquez, M. G. (2020). Proud but ashamed: narratives and moral emotions about the troubled national past in Spain. International Journal of Heritage Studies. doi: 10.1080/13527258.2020.1781680

Lowenthal, D. (1985). The past is a foreign country. Cambridge, MA: Cambridge University Press.

Lowenthal, D. (1998). The heritage crusade and the spoils of history. Cambridge, UK: Cambridge University Press.

Macintyre, S. y Clark, A. (2003). The History Wars. Melbourne: Melbourne University Press.

Maier, R. (2017). Tools in Teaching Recent Past Conflicts: Constructing Textbooks beyond National Borders. En M. Carretero, S. Berger, y M. Grever (eds.), Palgrave Handbook of Research in Historical Culture and Education (pp. 673-696). Londres: Palgrave Macmillan.

Olofsson, H., Samuelsson, J., Stolare, M. y Wendell, J. (2017). The Swedes and their history. London Review of Education, 15(2), 243-258. doi: 10.18546/LRE.15.2.08 
Podeh, E. (2000). History and memory in the Israeli educational system: The portrayal of the Arab-Israeli conflict in history textbooks (1948-2000). History and Memory, 12 (1), 65-100. doi: $10.1353 /$ ham.2000.0005

Popp, S. (2009). National textbook controversies in a globalizing world. En E. Erdmann, L. Cajani, A. S. Khodnev, S. Popp, N. Tutiaux-Guillon y G. D. Wrangham (eds.), History Teaching in the Crossfire of Political Interests (pp. 109-122). Schwalbach: Wochenschau.

Porat, D. (2001). A contemporary past: History textbooks as sites of national memory. En A. Dickinson, P. Gordon y P. Lee (eds.), Raising Standards in History Education (pp. 36-55). Londres: Woburn Press.

Renan, E. (1882). What is a Nation? En H. K. Bhabha (1990) (ed.), Nation and Narration (pp. 822). Londres: Routledge.

Repoussi, M. (2006). Politics questions history education: Debates on Greek history textbooks. En E. Erdmann, L. Cajani, A. S. Khodnev, S. Popp, N. Tutiaux-Guillon y G. D. Wrangham (eds.), Historical Consciousness - Historical Culture (pp. 99-110). Schwalbach: Wochenschau Verlag.

Rodríguez Moneo, M. y López, C. (2017). Concept Acquisition and Conceptual Change in History. En M. Carretero, S. Berger, y M. Grever (eds.), Palgrave Handbook of Research in Historical Culture and Education (pp. 469-490). Londres: Palgrave Macmillan.

Rüsen, J. (2004). Historical Consciousness: narrative structure, moral function, and ontogenetic development. En P. Seixas (ed.), Theorizing historical consciousness (pp. 63-85). Toronto: University of Toronto Press.

Sáiz, J. (2017). Pervivencias escolares de narrativa nacional española: Reconquista, Reyes Católicos e Imperio en libros de texto de historia y en relatos de estudiantes. Historia y Memoria de la Educación, 6, 165-201. doi: 10.5944/hme.6.2017.17137

Sáiz, J. (2018). Memorizar España sin pensarla históricamente: historia escolar e identidad nacional. En A. Delgado y A. Rivera (eds.), ¿Qué saben de su historia nuestros jóvenes? Enseñanza de la historia e identidad nacional (pp. 75-98). Granada: Comares.

Sáiz, J. y Barca, I. (2019). Narrativas nacionais de estudantes espanhóis e portugueses. Cadernos de Pesquisa, 49(172), 78-95. doi: 10.1590/198053145414

Sáiz, J. y López Facal, R. (2016). Narrativas nacionales históricas de estudiantes y profesorado en formación. Revista de educación, 374, 118-141. doi: 10.4438/1988-592X-RE-2016374-328

Sánchez, R., Arias, L. y Egea, A. (2016). The perduration of master narratives: The "discovery", conquest and colonisation of America in Spanish history textbooks. International Journal of Historical Learning, Teaching and Research, 13(2), 127-137.

Schissler, H. y Soysal, Y. N. (eds.) (2005). The nation, Europe, and the world: Textbooks and curricula in transition. Nueva York, NY.: Berghahn Books.

Seixas, P. (ed.) (2004). Theorizing historical consciousness. Toronto: University of Toronto Press.

Shemilt, D. (1980). Evaluation study: Schools council history 13-16 project. Edinburgh: Holmes McDougall.

Shepard, C., Reid, A. y Shepherd, K. (1993). Peace and war: Discovering the past Year 9. Londres: John Murray. 
Smith, A. D. (1991) National Identity. Londres: Penguin.

Symcox, L. y Wilschut, A. (eds.) (2009). National History Standards: The problem of the canon and the future of teaching history. Charlotte, NC: Information Age Publishing.

Van der Leeuw-Roord, J. (ed.) (2004). History changes. Facts and figures about history education in Europe since 1989. The Hague: EUROCLIO.

VanSledright, B. (2008). Narratives of nation-state, historical knowledge, and school history education. Review of Research in Education, 32(1), 109-146. doi: $10.3102 / 0091732 \times 07311065$

Voss, J. F. y Wiley, J. (2006). Expertise in History. En N. Ericsson, P. Feltovich y R. R. Hoffman (eds.), The Cambridge Handbook of Expertise and Expert Performance (pp. 569-584). Cambridge: Cambridge University Press.

Wertsch, J. V. (1997). Consuming Nationalism. Culture and Psychology, 3, 461-471. doi: $10.1177 / 1354067 \times 9734002$

Wertsch, J. V. (2002). Voices of Collective Remembering. Cambridge: Cambridge University Press.

Wertsch, J. V. (2017). Foreword to the special feature 'Negotiating the nation: Young people, national narratives and history education', edited by J. Létourneau and A Chapman. London Review of Education, 15(2), 152-154. doi: 10.18546/LRE.15.2.02

Wills, J. S. (2011). Misremembering as mediated action: Schematic narrative templates and elementary students' narration of the past. Theory \& Research in Social Education, 39(1), 115-144. doi: $10.1080 / 00933104.2011 .10473449$

Wineburg, S. (2001). Historical Thinking and Other Unnatural Acts. Filadelfia: Temple University Press. 


\section{Panta Rei}

PANTA REl es una revista digital de investigación orientada a la Historia y la Didáctica de la Historia. Su principal objetivo es la transmisión del conocimiento científico, dando una oportunidad también a los jóvenes investigadores que quieren abrirse camino en el estudio de las ciencias humanas y sociales. Se compone de estudios originales relacionados con la disciplina histórica así como su didáctica y difusión. Las diferentes secciones que componen la revista son: artículos de investigación, entrevistas a profesionales, recensiones de monografías de actualidad y crónicas de congresos o eventos científicos relevantes.

Todos los artículos publicados son objeto de un proceso de revisión a cargo de un mínimo de dos evaluadores, que se consideran expertos en el ámbito temático del artículo propuesto. Nuestro deseo es poder ofrecer unos contenidos rigurosos, de calidad y de interés.

El CEPOAT (Centro de Estudios del Próximo Oriente y la Antigüedad Tardía de la Universidad de Murcia) es la institución encargada de la coordinación y gestión de la revista, desde donde anualmente se lanzará la convocatoria para aquellos que estén interesados en publicar sus trabajos, siempre relacionados con la Historia y la Didáctica de la Historia.

PANTA REI is a digital journal focused on History and Teaching History. Its main objective is the transmission of scientific knowledge by giving also an opportunity to young researchers who want to make their way in the study of human and social sciences. It is composed by original studies related to History, as well as its didactics and promotion. The different sections of this journal are: research articles, interviews to professionals, recensions on monographs about current issues and reports about congresses or relevant scientific events.

All the articles published are subject to a revision process carried out by a minimum of two reviewers who are considered to be experts in the field of the article proposed. Our wish is to offer rigorous contents with quality and being of interest to the reader.

CEPOAT (Centre of Studies of the Middle East and Late Antiquity of the University of Murcia) is the institution in charge of the coordination and management of this journal. This is the centre from where the call for papers will be launched annually for all the people interested in publishing their papers, always related to History and Teaching History. 


\section{Normas de publicación}

El autor se compromete a enviar trabajos originales, que no se encuentren publicados en otras revistas ni en otros idiomas. Así mismo, el mismo artículo no podrá ser presentado en otras revistas mientras dure el proceso de evaluación.

\section{Envío y presentación de originales}

Las normas de edición y forma de envío de artículos a la revista se pueden consultar en https://revistas.um.es/pantarei/

Para la redacción de los trabajos se tendrá en cuenta el Manual de la American Psychological Association, en su $7 .^{a}$ edición. La extensión máxima de los trabajos será de 25 páginas. La revista acepta originales escritos en español o inglés.

\section{Proceso de valoración y evaluación}

Una vez recibidos los trabajos, la Revista realizará una primera valoración. Si el trabajo enviado se ajusta a las normas de presentación propuestas, la temática es coincidente con la línea editorial de la revista y posee la calidad científica necesaria, será remitido al consejo asesor para una primera evaluación. Si no es así en este primer paso se puede rechazar directamente los documentos que incumplan claramente la línea editorial.

Será el Consejo Asesor quien indique a la revista la originalidad, relevancia, estructura, redacción, aparato bibliográfico, etc. del trabajo enviado y, para ello, se designará a dos revisores expertos externos que evaluarán cada uno de los trabajos, que pueden formar parte (o no) de este Consejo Asesor. La selección de los revisores se ajustará a la temática y características metodológicas del trabajo. El nombre y filiación de los autores serán eliminados del trabajo para su revisión, así como los revisores actuarán de manera anónima y confidencial.

Los revisores deberán rellenar un informe de evaluación que centrará su atención en aspectos tales como características formales, originalidad y novedad de los trabajos, relevancia de las propuestas y los resultados, calidad metodológica y validez científica.

Una vez terminado el proceso se decidirá la aceptación o no de los mismos y su publicación en el número que sea pertinente, así como las modificaciones susceptibles de ser realizadas para su final publicación. Dicha notificación se enviará únicamente por correo electrónico, en un plazo máximo de seis meses. 


\section{Publishing rules}

The author is committed to submit original papers not having been published in other reviews or in other languages. In this way, it is not allowed for the same paper to be presented in other reviews during the evaluation process.

\section{Submission and presentation of originals}

The editing rules and the guidelines for the submission of papers can be consulted at https://revistas.um.es/pantarei/

The seventh edition of the Manual of the American Psychological Association will be taken into account for the writing of the papers. The length of the submitted papers will not exceed the 25 pages. The journal accepts originals written in Spanish or English.

\section{Examination and assessment process}

The Journal will submit the papers to a first examination once received. If the paper follows the presentation guidelines, the subject agrees with the editorial line of this journal, and possess the scientific quality required, it will be sent to the advisory council for a first assessment. If not, the documents which clearly fail to complete the editorial line may be rejected straightaway in this first step.

The Advisory Council will indicate the originality, relevance, structure, writing, bibliography, etc. of the text to the journal; for this purpose, two outside experts will be designated to review the papers; these experts can be (or not) part of this Advisory Council. The selection of the experts will adjust to the subject and methodological characteristics of the paper. Name and affiliation of the author will be eliminated from the text for its review, in this way experts will act anonymously and confidentially.

The experts will fill out an assessment report which will focus on aspects such as formal characteristics, originality and novelty of the papers, relevance and results of the proposal, methodological quality and scientific validity.

Once the process is finished, the acceptance or not of the papers and its publication in the corresponding edition will be decided, as well as the modifications that may be done for its final publication. This notification will be sent by email within 6 months maximum. 


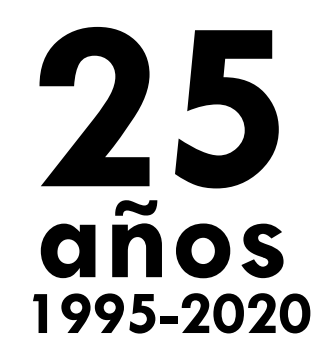

\section{cepoAt editum}

